\title{
Phenotypic flexibility as key factor in the human nutrition and health relationship
}

\author{
Ben van Ommen • Jan van der Greef • \\ Jose Maria Ordovas $\cdot$ Hannelore Daniel
}

Received: 22 May 2014/Accepted: 25 July 2014/Published online: 9 August 2014

(C) The Author(s) 2014. This article is published with open access at Springerlink.com

\begin{abstract}
Metabolic adaptation to a disturbance of homeostasis is determined by a series of interconnected physiological processes and molecular mechanisms that can be followed in space (i.e., different organs or organelles) and in time. The amplitudes of these responses of this "systems flexibility network" determine to what extent the individual can adequately react to external challenges of varying nature and thus determine the individual's health status and disease predisposition. Connected pathways and regulatory networks act as "adaptive response systems" with metabolic and inflammatory processes as a core-but embedded into psycho-neuro-endocrine control mechanisms that in their totality define the phenotypic flexibility in an individual. Optimal metabolic health is thus the orchestration of all mechanisms and processes that maintain this flexibility in an organism as a phenotype. Consequently, onset of many chronic metabolic diseases results from impairment or even loss of flexibility in parts of the system. This also means that metabolic diseases need to be
\end{abstract}

B. van Ommen $(\bowtie) \cdot$ J. van der Greef

TNO, Zeist, The Netherlands

e-mail: ben.vanommen@tno.nl

J. van der Greef

Leiden University, Leiden, The Netherlands

J. van der Greef

Sino-Dutch Centre for Preventative and Personalized Medicine,

Zeist, The Netherlands

J. M. Ordovas

Nutrition and Genomics Laboratory, Tufts University Boston,

Boston, MA, USA

H. Daniel

Technical University Munich, Munich, Germany diagnosed and treated from a systems perspective referring to a "systems medicine" approach. This requires a far better understanding of the mechanisms involved in maintaining, optimizing and restoring phenotypic flexibility. Although a loss of flexibility in a specific part of the network may promote pathologies, this not necessarily takes place in the same part because the system compensates. Diagnosis at systems level therefore needs the quantification of the response reactions of all relevant parts of the phenotypic flexibility system. This can be achieved by disturbing the homeostatic system by any challenge from extended fasting, to intensive exercise or a caloric overload.

Keywords Phenotypic flexibility - Systems biology · Metabolism · Biomarker

\section{Introduction}

Physiology maintains a well-orchestrated machinery allowing the organism to adapt to the continuously changing environment, of which food takes a major share. We term this organism response capacity "phenotypic flexibility" and this is from our understanding central for overall homeostasis and thus for a healthy life. Processes and mechanisms forming the basis of phenotypic flexibility include substrate fluxes for ATP production and biosynthesis, oxidative stress and inflammatory responses, immune functions as well as DNA repair, apoptosis and others. All of these processes involve multiple molecular mechanisms, interconnected and coordinated by complex regulatory networks which need a real systems approach for the most comprehensive description and in-depth understanding. 
Daily meals provide energy pulses which are efficiently absorbed and distributed for immediate needs in ATP production or stored as glycogen or lipids. Possible side effects of these energy pulses are oxidative and inflammatory responses that are ideally quenched by counteracting mechanisms. Stress reactions on the other side require the immediate mobilization of endogenous energy resources with changes in substrate flow for the metabolic needs. Chronic stress conditions may induce adaptation processes that go beyond the limits of normal phenotypic flexibility leading to progressive inflexibility, which in turn contributes to a disease onset. Food components, by excess or by lack in the diet, challenge phenotypic flexibility: Essential nutrients and other food bioactives, when consumed appropriately, play key roles in the mechanisms that maintain phenotypic flexibility, for example as cofactors, while excess of energy, high glucose and fructose intakes or certain trans-fatty acids cause a decline in phenotypic flexibility.

The importance of maintaining flexibility as a key feature to optimal health calls for new research on the relationship between nutrition and health and in particular with the identification of valid "biomarkers of health," related to the dynamics of regulatory processes. These markers may be found by exploiting the processes and quantify the response reactions to a stress challenge including a "dietary stress" (van Ommen et al. 2008, 2009).

The key example for the loss of phenotypic flexibility is the development of type 2 diabetes with the impaired response to insulin secretion. It usually takes years from an initial impairment of insulin action in peripheral tissues and liver with hyperglycemia, hyperinsulinemia to a progressive loss of $\beta$-cell responsiveness and finally complete loss of $\beta$-cell mass. Proper diagnosis of phenotypic flexibility in this context can be made by analyzing all relevant changes in accessible compartments (plasma, exhaled air, urine) during an oral glucose tolerance test (OGTT) and define the amplitude of the changes over time in healthy individuals and those with insulin resistance or diabetes (Ho et al. 2013). But this example also illustrates the complex nature of the phenotypic flexibility concept. Hepatic insulin resistance may be caused by fatty liver as a result of adipose tissue insulin resistance with a limited capacity of adipocytes to take up glucose for conversion into fatty acids and triglycerides (Virtue and Vidal-Puig 2010). But hepatic lipid accumulation may also be caused by a reduced biosynthesis of phosphatidylcholine that is needed for export of the lipids via VLDL and/or by an impaired hepatic fatty acid oxidation (Vance 2013). In view of other dietary determinants, a high fructose intake also increases hepatic de novo fatty acid biosynthesis and the development of non-alcoholic fatty liver disease. In contrast to liver, insulin resistance in peripheral tissues is not only caused by lipotoxicity but most likely also involves, in a causative manner, alterations in amino acid homeostasismainly of the branched chain amino acids-and overall protein homeostasis.

Phenotypic flexibility presents a systems view on the complete molecular and physiological "machinery" of stress responses related to metabolic and caloric challenges (Fig. 1). The paragraphs below illustrate the concept of phenotypic flexibility by describing the human phenotypic responses to the three macronutrients glucose, triglycerides and proteins. Together, these examples span from intracellular regulatory mechanisms to whole-body physiology and show the complexity and the need for a systems approach in studying phenotypic flexibility and its application in metabolic health research and systems healthcare.

\section{Phenotypic flexibility responses to a glucose challenge}

The signaling compensatory response on glucose

Our metabolic system appears not 'designed' to deal with large amounts of simple carbohydrate, although dietary intake of large quantities of complex carbohydrates (starch) has been part of human diets throughout recent evolution. Except for some fruits and honey delivering mono and disaccharides in early phases of human evolution, regular consumption of higher amounts of mono- and disaccharides with foods and beverages has become prevalent only in the last few decades. Glucose release from complex carbohydrates such as starch is rate limiting primarily because hydrolysis of glucosidic bonds occurs in the upper small intestine by pancreatic amylase. In contrast, administration of $75 \mathrm{~g}$ of D-glucose as during a standard OGTT causes an immediate increase in plasma glucose levels and triggers a large array of compensatory mechanisms. Gastrointestinal hormones such as CCK (Geneontology), GI, GLP1 and PYY increase in response to OGTT (Blaak et al. 2012), which may reflect an "archaic response" of the gastrointestinal tract to a complex meal containing fat, carbohydrates and proteins rather than the artificial condition of an isolated nutrient such as glucose entering the system. Increased plasma glucose, together with the elevated levels of the incretins GIP and GLP1, causes insulin and C-peptide secretion from pancreatic $\beta$-cells and a modest inhibition of glucagon secretion from $\alpha$-cells. Insulin controls glucose disposition in muscle and adipose tissue by recruitment of the glucose transporter GLUT4 into the plasma membrane. The presence of additional GLUT4 allows increased glucose uptake. In the liver, the additional glucose induces glycogen production by changing the activity states of glycogen synthase and phosphorylase downstream of the insulin receptor. 


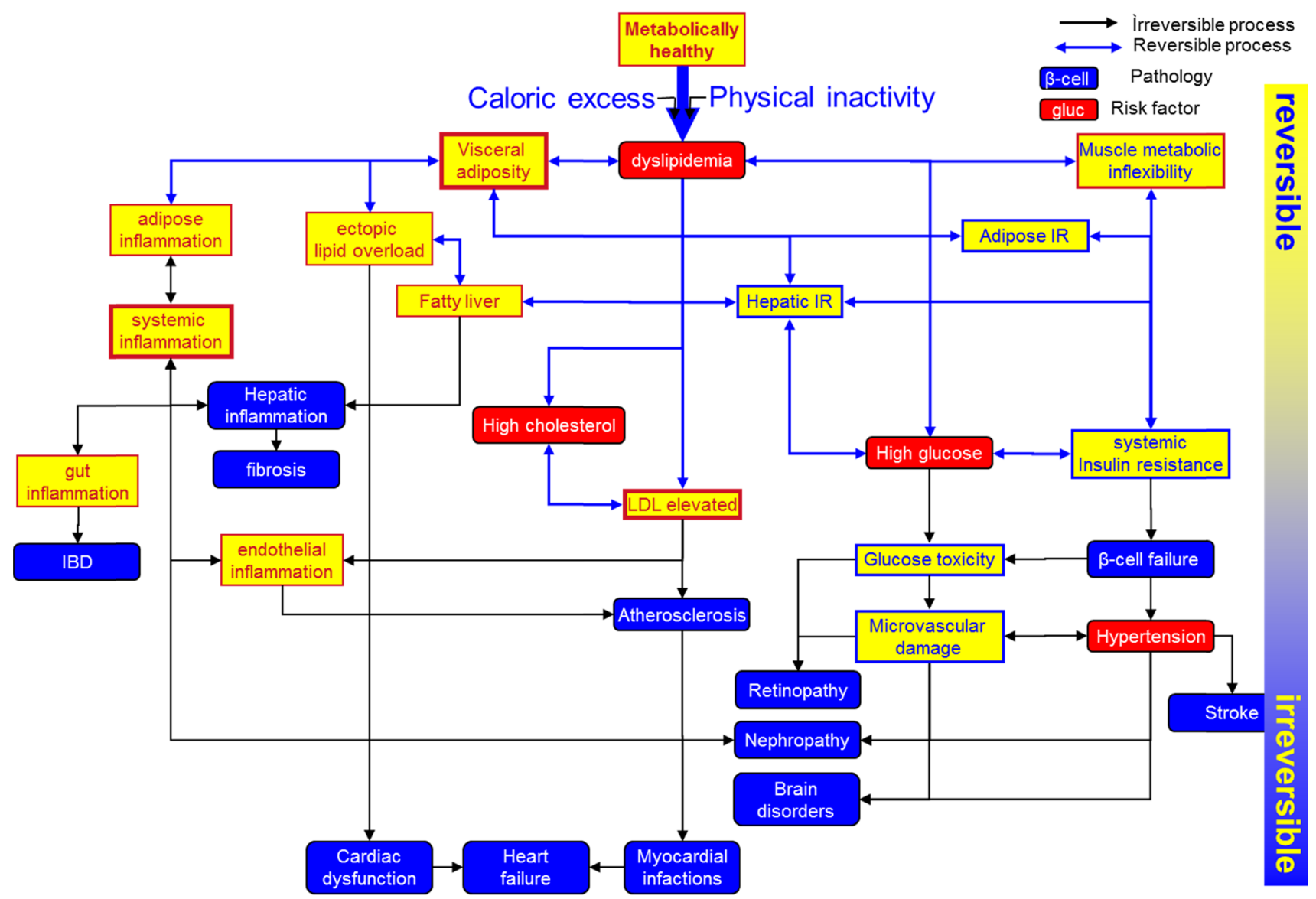

Fig. 1 Main processes and events involved in (decrease of) phenotypic flexibility and the resulting (patho-)physiological consequences (adapted from Lusis et al. 2008). Multiple organs and processes are involved in maintaining homeostasis in aspects of metabolism, all of them are interacting with each other and most of them are reversible

to protein synthesis which depends on insulin receptor and mTOR activation. Interestingly, almost all the amino acids that show decreased plasma levels in response to OGTT have been identified in cohort studies as "marker metabolites" of insulin resistance and type 2 diabetes mellitus with increased levels in fasting state (Newgard et al. 2009). Others have associated changes in plasma levels of some of these amino acids as predictive for disease development. This emphasizes the key role of insulin not only in glucose handling but also in amino acid metabolism.

The OGTT in addition causes a suppression of lipolysis that in turn reduces plasma non-esterified fatty acids (NEFA) and ketone body concentrations. Elevated levels of those metabolites in fasting blood also characterize an insulin-resistant state and type 2 diabetes. Interestingly, there are also significant reductions in plasma acyl-carnitine levels, while free carnitine increases (Krug et al. 2012). Since acyl-carnitines are the products of mitochondrial $\beta$-oxidation, these changes in plasma during the OGTT demonstrate the shift from utilization of fatty acids as prime energy substrates during fasting to glucose 
prolonged fasting

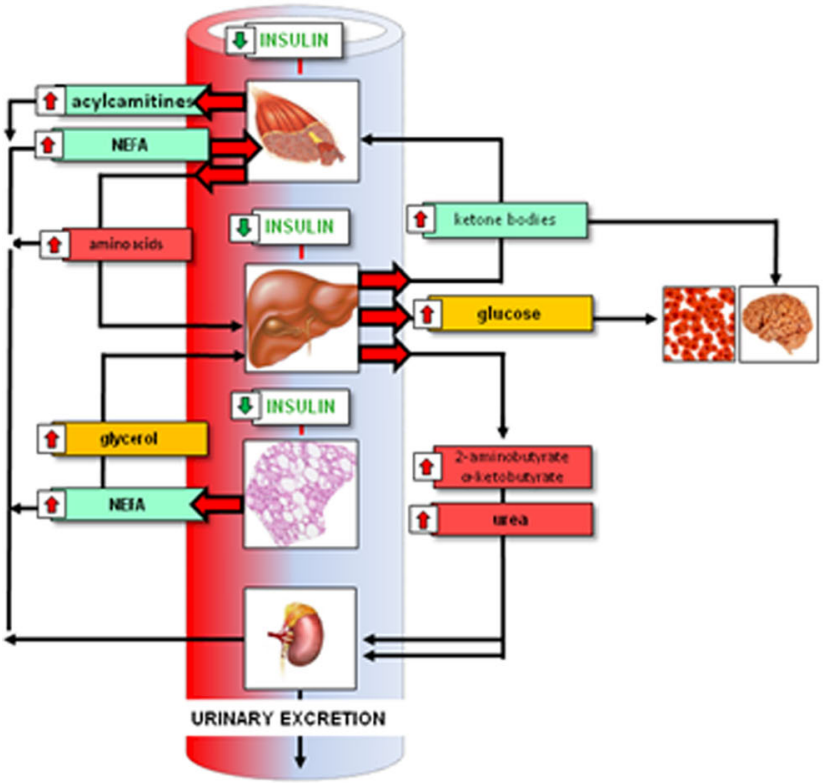

Fig. 2 Whole-body metabolic condition and control circuits characterizing a catabolic (fasting) as compared to anabolic/postprandial state with major changes in hormone and metabolite levels which

utilization following the OGTT. After at least $3 \mathrm{~h}$, plasma levels of all measured metabolites have returned to baseline and a steady increase in plasma NEFA, ketone bodies, and amino acid levels occurs again. Those changes, however, are delayed in humans with hyperinsulinemia and insulin resistance indicating a prolonged stay in an anabolic state. In this respect, the delay in clearance of plasma glucose is associated with a delayed return of catabolic marker metabolites to initial values. These changes in plasma metabolite patterns occur in essentially all major metabolic pathways of macronutrient metabolism (glucose, fatty acids, amino acids). Since the release and uptake of these metabolites by organs depend on insulin sensitivity, the OGTT seems particularly suitable to assess phenotypic flexibility of individuals in time-dependence and in organ specificity (Fig. 2).

Fasting and flexibility

In contrast to the postprandial state, fasting is characterized by a flow of free fatty acids from adipose tissue to muscle and liver, an increase in hepatic ketone body production, and a release of amino acids into circulation mainly for use in hepatic gluconeogenesis. The OGTT, usually performed after an overnight fasting period, reverses these processes within 15-30 min. The time-dependent response and return to homeostasis to an OGTT thus may be taken as a measure of the individual's flexibility to switch from a catabolic to oral glucose tolerance test

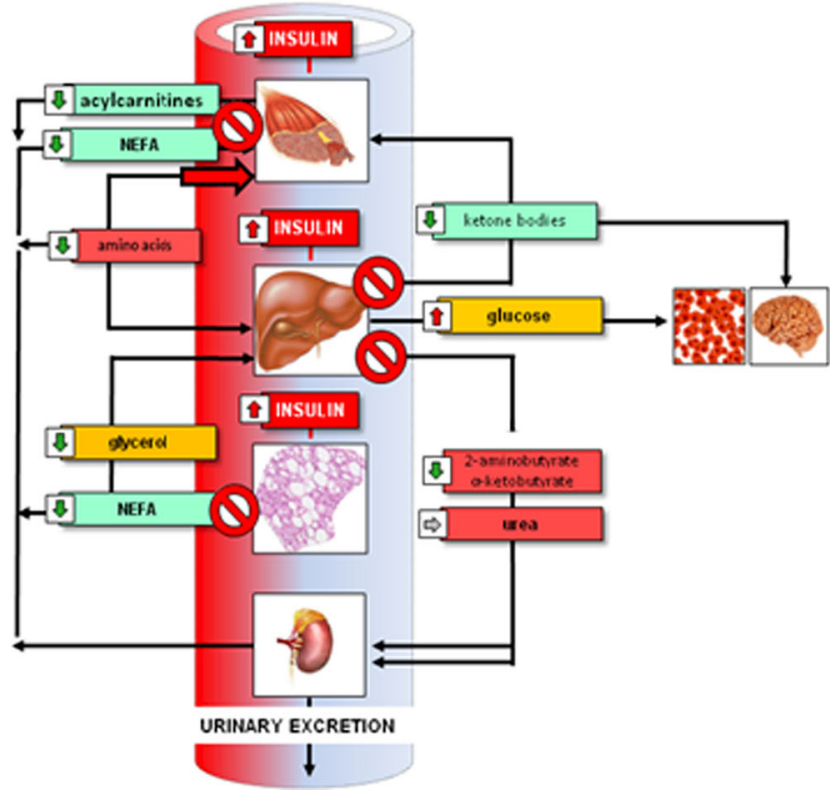

define the etches of a metabolic space and in their time-dependence also serve as a measure of phenotypic flexibility

an anabolic state. Recent studies employing plasma metabolite profiling during the fasting state (Lawton et al. 2008) and after an OGTT in humans (Shaham et al. 2008; Wopereis et al. 2009; Zhao et al. 2009; Spégel et al. 2009; Ho et al. 2013; Krug et al. 2012) have revealed major changes in numerous plasma metabolites that go far beyond the well-known changes in plasma glucose and insulin or NEFA levels.

\section{Phenotypic flexibility responses to triglyceride challenges}

The liver as gatekeeper in a triglyceride challenge

The hydrolysis of triglycerides in the intestinal lumen and absorption of the constituents is efficient. Lipids are assembled into chylomicrons with the help of apolipoproteins synthesized in epithelial cells and are released into lymph. Because the intestine expresses many enzymes that control mitochondrial, peroxisomal and microsomal oxidation, fatty acids can also be utilized by the enterocyte. Fatty acids play an important role as signaling molecules in the regulation of their own metabolism. The transcription factor PPAR $\alpha$, a member of the superfamily of nuclear receptors, plays an essential role in this regulation. The liver is the master gatekeeper of ingested and de novo synthesized lipids, with far greater capacity than the 
intestine for storage and maintenance of lipid homeostasis during the transition from fed to fasted states. Both organs, through the assembly and secretion of lipid-containing particles, deliver lipids in the form of lipoproteins to peripheral tissues and cycle between postprandial and postabsorptive states. Oral fat challenges showed dramatic inter-individual differences between the metabolism of triglyceride-rich lipoproteins, attributed to age, sex, genetic factors but also to the characteristic of the fat load or the background diet (Fielding 2011).

Plasma clearance as flexibility parameter

Importantly, the observed associations between postprandial lipemia-related variables and CVD risk are not primarily due to the levels of chylomicrons released by the gut into the circulation, but rather to the levels of chylomicron remnants remaining in circulation following the fat load and the time required for their total clearance. Apparently, the capacity (flexibility) of the system to return to equilibrium after a metabolic challenge is more relevant to health than the actual deviation from the steady state. Therefore, an impaired catabolism of chylomicron remnants may represent early changes in phenotypic flexibility given their association with cardiovascular disease risk (Chan et al. 2013; Mihas et al. 2011). Besides the measurement of chylomicron remnants or APOB48 (Fujioka and Ishikawa 2009), more recent studies have described the time courses of plasma metabolites and proteins upon different scenarios involving dietary challenges (Beaudoin et al. 2011). However, the relation between traditional and novel biomarkers has not been established.

\section{Adipose expandability}

Following the packaging of dietary fat into chylomicrons and release into lymph, a key tissue for its disposal is adipose tissue and a fundamental element in phenotypic flexibility of the adipose tissue is its expandability. The failure in proper adipose tissue expansion rather than obesity per se is a key factor by which a positive energy balance is linked to type 2 diabetes as well as other metabolic diseases (Virtue and Vidal-Puig 2010; Medina-Gomez et al. 2007). Once the individual's capacity to store additional fat in the adipocyte is reached, lipids begin to accumulate in other tissues such as liver and muscle leading to harmful consequences (Virtue and Vidal-Puig 2010).

\section{Circadian rythm}

The circadian rhythm is one of the best established mechanisms of phenotypic flexibility that has been ingrained in all living things in order to maintain synchronicity with the pace defined by the rotation of the Earth around its axis. Based on data from studies determining the circadian metabolomic changes, it has become evident that the lipid metabolome is among the most modulated pathways, underscoring the importance of lipid metabolism, including dietary fat and relevance of maintaining this pathway attuned with the environmental changes driven by the daily rhythms of nature. (Eckel-Mahan et al. 2012; Dallmann et al. 2012).

The inflammatory response

A postprandial inflammatory response to triglicerides (Giuseppe Derosa et al. 2009) but also to glucose (Derosa et al. 2010) or a mixed meal challenge has been described. Yet, a thorough nutrigenomic examination of the inflammatory response resulted in only subtle and minor effects (Pellis et al. 2011; Wopereis et al. 2013). Apparently, in healthy subjects, phenotypic flexibility mechanisms prevent the onset of a strong inflammatory reaction. Interestingly, many studies reporting on a postprandial inflammatory response had no proper control group, confounding the conclusions (for a discussion see (Wopereis et al. 2013). Yet, this area needs further attention, as many relevant but controversial mechanisms have been identified in animal models but still need to be validated for human translation, such as lipid-based leaky gut systemic LPS inflammation (Liang et al. 2014), differences between saturated and unsaturated hepatic inflammation triggers (Lichtenstein et al. 2010). Obviously, chronic caloric overload produces a metabolic-inflammatory response of which the mechanisms are being unraveled (Osborn and Olefsky 2012), but the acute inflammatory response needs further attention as the molecular tools to study this become available.

\section{Phenotypic flexibility responses to protein challenges}

The importance of amino acid homeostatic control

Dietary protein intake has been fundamentally important for the development of the human species because the amino acid substrates for protein synthesis do not have to be synthesized de novo. Increased protein intake not only allowed the body length to increase in human evolution but was also a key determinant in brain development (Laus et al. 2011). Yet, as protein synthesis cannot be increased infinitely, the current luxury supply of dietary protein requires that substantial quantities of amino acids are oxidized and used as energy substrates. Amino acids contribute on average with 15 to $20 \%$ to fuel oxidation. 
However, a consequence of amino acid oxidation is the increased release of ammonia which needs to be detoxified by the hepatic urea cycle followed by elimination through the kidneys. In addition, the surplus of methionine and cysteine in the diet provided in larger quantities with animal protein sources increases of sulfate excretion via kidney with net effects on extracellular acidification and increased renal acid elimination. The daily turnover of amino acids exceeds that of lipids by around $30 \%$ and is almost two-fold higher than that of glucose.

Plasma homeostasis of amino acids is most important because numerous individual amino acids have distinct metabolic roles such as neurotransmitters (i.e., glutamate, glycine) or precursors thereof (GABA from glutamate, or catecholamines from tyrosine) or contribute to a variety of synthetic pathways or other regulatory processes. A prime example is nitric oxide (NO) which is derived from arginine and acts as a signaling molecule in numerous processes (Hoang et al. 2013). It has been proposed that dietary arginine is converted into citrulline in the intestine which prevents arginine plasma levels to overshoot in the absorptive phase which would lead to an increased NO production with numerous downstream effects for example on the vasculature (Kaore et al. 2013). Only small quantities of portal blood citrulline are taken up during liver passage, but the kidney extracts most citrulline and converts it back to arginine. Liver extraction of other amino acids delivered through portal blood is substantial with the exception of the branched chain and aromatic amino acids that consequently show the most impressive increases in peripheral blood after protein intake.

\section{Signaling control of amino acid homeostasis}

Amino acid homeostasis is thus tightly regulated at all levels (absorptive, anabolic, catabolic, protein synthesis or degradation). Protein turnover as the sum of degradation and synthesis is subject to regulation by numerous hormones. Insulin reduces protein breakdown and promotes protein synthesis when plasma insulin levels rise and amino acid levels also increase following protein intake. Under these conditions, insulin has the strongest effects on protein balance compared to all other hormones including growth hormone/IGF, epinephrine, testosterone or thyroid hormones. In contrast to insulin, stress hormones such as glucagon, glucocorticoids and catecholamines strongly increase protein breakdown but seem also to be able to modestly increase protein synthesis rate.

In contrast to carbohydrate-rich meals, protein-rich meals cause glucagon secretion, which also occurs in the presence of dietary carbohydrates with a simultaneous increase in plasma insulin. Although some amino acids can effectively elicit insulin secretion (for example arginine), the totality of circulating amino acids in the absorptive phase promotes a substantial glucagon release. This counteracting effect of amino acid on insulin secretion prevents a hypoglycemia during high protein, but low carbohydrate intake. Glucagon increases hepatic gluconeogenesis from amino acids allowing under high protein intake the maintenance of plasma glucose levels and reserves of glycogen. Yet, the clearance of the amino acids from plasma in the postprandial state is increased in the presence of insulin. In diabetes, removal of amino acids from plasma seems delayed and gluconeogenesis from amino acids in liver seems enhanced by the lack of proper suppression by insulin, thus contributing to hyperglycemia. In view of the effects of insulin on protein synthesis in the presence of elevated plasma amino acids during high protein intake, the concerted action of the insulin and mTOR signaling comes into play. mTOR comprises two protein complexes TORC1 and TORC2. mTORC1 (mammalian TORC1) functions as an environmental sensor to promote anabolic and inhibit catabolic cellular functions. It phosphorylates and activates protein kinases S6K1 and S6K2 with the substrate ribosomal protein S6, a component of the $40 \mathrm{~S}$ ribosome and of $4 \mathrm{E}-\mathrm{BP} 1$ promoting ribosome genesis and protein synthesis. Intracellular amino acids and among them leucine are sensed by GTPases and activate mTORC1. The downstream target of the insulin receptor signaling pathway Akt inhibits the TSC2 protein and allows Rheb-GTP to accumulate and to activate mTORC1 and thus amplifies the amino acid dependent activation. On the other hand, AMP-kinase is a sensor of the cellular energy status and exerts inhibitory effects on the mTOR system. Interestingly, exercise also enhances mTOR activity and thus promotes metabolic adaptation to nutrients and exercise in skeletal muscle. However, mTORC1 signaling can attenuate Akt activation through a negative feedback mechanism. Inhibitory phosphorylation of the insulin receptor substrate 1 and the mTORC2 component Rictor by mTORC1 and S6-kinase suppresses the ability of insulin to stimulate Akt as a key effector of insulin signaling. Amino acid induced mTORC1 signaling can decrease insulin sensitivity, and this can be observed in all metabolically important tissues examined. In this respect, it is not surprising that amino acid homeostasis is impaired in humans with the metabolic syndrome. That obesity affects amino acid metabolism is known since 1969 when it was first described that plasma levels of branched chain amino acids (BCAA) are elevated in obesity (Felig et al. 1969). It was recently shown that these changes in plasma BCAA are also found in obese children and that they predict future development of insulin resistance (McCormack et al. 2013). Numerous studies employing metabolite profiling in insulin-resistant humans or patients with type 2 diabetes (fasting plasma) have identified predominantly amino acids 
such as the BCAA and their degradation products (Newgard et al. 2009), glycine, citrulline and proline but also aminoadipic acid from lysine degradation as marker metabolites (Menni et al. 2013). However, there are currently no studies that demonstrate impairments in phenotypic flexibility related to amino acid or nitrogen handling in insulin resistance or diabetes when the metabolic system is challenged with larger quantities of dietary protein.

Taken together, a high protein intake-whether acute or chronic-has complex effects on the metabolic system. It reduces the metabolic adaptation to insulin action, and forces amino acids to be used as energy substrates thus reducing the need to burn lipids and carbohydrates which may promote insulin resistance and diabetes by their glucotoxicity and lipotoxicity. In addition, increased gluconeogenesis in insulin-resistant state further enhances hyperglycemia. The need for elimination of the nitrogen surplus challenges hepatic urea production and the renal elimination. In addition, sulfur derived from methionine and cysteine intake challenges the acid-base balance.

\section{Conclusion and perspective}

The above examples of carbohydrate (glucose), lipid (triglycerides and fatty acids) and protein related phenotypic flexibility all trigger acute and complex stress response mechanisms of which optimal functioning is essential for maintenance of metabolic health. Quantification of aspects of these stress response reactions is informative on health status and can be exploited as "biomarker of health." In this sense, the OGTT is well established and discussed above, but research on triglycerides (Blaak et al. 2006; Krug et al. 2012; Musso et al. 2009), protein (Fernandes and van de Kamer 1968; Bosch et al. 1984) and various combinations of glucose, triglycerides and proteins, either as mixture of the pure components (Pellis et al. 2011; Wopereis et al. 2013) or as mixed meal challenges (Phillips et al. 2010; van Dijk et al. 2012) has been published. Also, mathematical methods to analyze the stress response curves are being developed (Vis et al. 2014). Each of these "stress tests" has pro's and con's in terms of practical use vs value for mechanistic interpretation. Apart from the OGTT, the "standardized phenotypic flexibility assay" now emerges as $75 \mathrm{~g}$ of glucose, $60 \mathrm{~g}$ of Palm oil and $30 \mathrm{~g}$ of protein.

All of these acute response reactions illustrate a number of characteristics of phenotypic flexibility:

1. All processes result from an acute perturbation and show a time course of stress response in the hour scale, at first glance simple ("glucose curve") but intrinsically complicated, spanning major parts of metabolicinflammatory control;
2. All processes are interdependent and can act via multiple connections and feedback mechanisms;

3. Failure of a specific process in this phenotypic flexibility network may be compensated by other processes, but eventually lead to harmful adaptive processes;

4. Interestingly, the "site of failure" does not need to be the site of emerging pathology. Impaired adipose expandability will result in ectopic lipid, inflammation and hepatic/vascular disease.

5. Quantification of the molecular stress response reactions after a standardized metabolic/caloric perturbation such as the OGTT (Krug et al. 2012), standardized mixed meal challenges (Pellis et al. 2011) or even Big Macs (Bondia-Pons et al. 2014) (e.g., by plasma metabolomics) reveals a detailed picture of the events and can be used as biomarker and diagnostics of phenotypic flexibility.

In contemplating health from a phenotypic flexibility systems perspective, prevention of disease onset and the role of diet in maintaining health become tangible and concrete as many nutrients play a crucial role in the mechanisms underlying the response pathways. For example, dietary factors such as fructose but also betaine, choline, carnitine and others play an important role both in onset and prevention of fatty liver (de Wit et al. 2012; Malaguarnera et al. 2010; Noland et al. 2009; Rutlegde and Adeli 2007; Fischer et al. 2010). Oxidative stress and inflammatory stress responses also rely on diet-derived factors such as the eicosanoids as products of essential fatty acids (Wopereis et al. 2013). We now realize that, in general, dietary factors have roles that are mirror images of those provided by drugs: The first optimize and facilitate processes (salutogenesis, health promotion), while the latter mainly inhibit processes (disease management), which by definition does not match with the concept of strengthening or improving phenotypic flexibility.

Finally, we would like to emphasize that phenotypic flexibility as defined above is embedded in a larger system. It is part of a much wider flexibility system determining and maintaining optimal health, which can only be understood by integrating physiology and psychology, and also taking into account the social environment. This biopsychological domain focuses on psycho-neuro-endocrinology instead of neuro-endocrinology alone. Visceral adiposity is not only associated with a blunted metabolic flexibility, but also with reduced flexibility in neuroendocrine and cognitive responses (Jones et al. 2012). From the psychological perspective, the relationship with metabolic/ inflammatory health is becoming apparent. For example, social support is related to changes in cardiovascular, neuroendocrine and immune function, optimism has been 
associated with immune up-regulation, and adaptive coping is linked with reduced cortisol output (see (Steptoe et al. 2009) and references). Here, the well-known concept in psychology of resilience nicely aligns with phenotypic flexibility.

Secondly, flexibility has different time dimensions. As described above, phenotypic flexibility responses to acute challenges occur with timescales of minutes to hours while chronic adaptation processes and allostatic processes, as the consequence of all efforts to maintain homeostasis and flexibility that result in pathologies, usually span months to decades. Some of these adaptive processes and their health consequences have already been mentioned above.

The emphasis for life science research has to be aligned with the improved insights related to the new concepts and definitions of health. Studies need to focus more on system response monitoring, measuring the dynamics of regulatory processes in a much broader context.

Acknowledgments Part of this work has been funded by the FP7 project NutriTech. NutriTech is financed by the European Commission in the 7th Framework Programme FP7, Grant agreement no: 289511 Version date: 2012-11-30.

Open Access This article is distributed under the terms of the Creative Commons Attribution License which permits any use, distribution, and reproduction in any medium, provided the original author(s) and the source are credited.

\section{References}

Beaudoin MS, Robinson LE, Graham TE (2011) An oral lipid challenge and acute intake of caffeinated coffee additively decrease glucose tolerance in healthy men. J Nutr 141(4):574-581. doi:10.3945/jn.110.132761

Blaak EE, Hul G, Verdich C, Stich V, Martinez A, Petersen M, Feskens EFM et al (2006) Fat oxidation before and after a high fat load in the obese insulin-resistant state. J Clin Endocrinol Metab 91(4):1462-1469. doi:10.1210/jc.2005-1598

Blaak EE, Antoine J-M, Benton D, Björck I, Bozzetto L, Brouns F, Diamant $M$ et al (2012) Impact of postprandial glycaemia on health and prevention of disease. Obes Rev 13(10):923-984. doi:10.1111/j.1467-789X.2012.01011.x

Bondia-Pons I, Maukonen J, Mattila I, Rissanen A, Saarela M, Kaprio J, Hakkarainen A et al (2014) Metabolome and fecal microbiota in monozygotic twin pairs discordant for weight: a big mac challenge. FASEB J, 1-11. doi:10.1096/fj.14-250167

Bosch JP, Lauer A, Glabman S (1984) Short-term protein loading in assessment of patients with renal disease. Am J Med 77(5):873-879

Chan DC, Pang J, Romic G, Watts GF (2013) Postprandial hypertriglyceridemia and cardiovascular disease: current and future therapies. Curr Atheroscler Rep 15(3):309. doi:10.1007/ s11883-013-0309-9

Dallmann R, Viola AU, Tarokh L, Cajochen C, Brown SA (2012) The human circadian metabolome. Proc Natl Acad Sci USA 109(7):2625-2629. doi:10.1073/pnas.1114410109

De Wit NJW, Afman LA, Mensink M, Müller M (2012) Phenotyping the effect of diet on non-alcoholic fatty liver disease. J Hepatol $\operatorname{xxx}$ (6). European Association for the Study of the Liver: 1370-73. doi:10.1016/j.jhep.2012.07.003

Derosa G, Ferrari I, D’Angelo A, Salvadeo SAT, Fogari E, Gravina A, Mereu R et al (2009) Oral fat load effects on inflammation and endothelial stress markers in healthy subjects. Heart Vess 24(3):204-210. doi:10.1007/s00380-008-1109-y

Derosa G, D'Angelo A, Salvadeo SAT, Ferrari I, Fogari E, Gravina A, Mereu R et al (2010) Oral glucose tolerance test effects on endothelial inflammation markers in healthy subjects and diabetic patients. Horm Metab Res 42(1):8-13. doi:10.1055/s0029-1237728

Eckel-Mahan KL, Patel VR, Mohney RP, Vignola KS, Baldi P, Sassone-Corsi P (2012) Coordination of the transcriptome and metabolome by the circadian clock. Proc Natl Acad Sci USA. doi:10.1073/pnas.1118726109

Felig P, Marliss E, Cahill GF (1969) Plasma amino acid levels and insulin secretion in obesity. N Eng J Med 281(15):811-816. doi:10.1056/NEJM196910092811503

Fernandes J, van de Kamer JH (1968) Hexose and protein tolerance tests in children with liver glycogenosis caused by a deficiency of the debranching enzyme system. Pediatrics 41(5):935-944

Fielding B (2011) Tracing the fate of dietary fatty acids: metabolic studies of postprandial lipaemia in human subjects. Proc Nutr Soc 70(3):342-350. doi:10.1017/S002966511100084X

Fischer LM, da Costa KA, Galanko J, Sha W, Stephenson B, Vick J, Zeisel SH (2010) Choline intake and genetic polymorphisms influence choline metabolite concentrations in human breast milk and plasma. Am J Clin Nutr 92(2):336-346. doi:10.3945/ ajcn.2010.29459

Fujioka Y, Ishikawa Y (2009) Remnant lipoproteins as strong key particles to atherogenesis. J Atheroscler Thromb 16(3):145-154

Geneontology. http://amigo.geneontology.org/

Ho JE, Larson MG, Vasan RS, Ghorbani A, Cheng S, Rhee EP, Florez JC, Clish CB, Gerszten RE, Wang TJ (2013) Metabolite profiles during oral glucose challenge. Diabetes 62(8):2689-2698. doi: $10.2337 / \mathrm{db} 12-0754$

Hoang HH, Padgham SV, Meininger CJ (2013) L-Arginine, tetrahydrobiopterin, nitric oxide and diabetes. Curr Opin Clin Nutr Metab Care 16(1):76-82. doi:10.1097/MCO.0b013e32835ad1ef

Jones A, McMillan MR, Jones RW, Kowalik GT, Steeden JA, Deanfield JE, Pruessner JC, Taylor AM, Muthurangu V (2012) Adiposity is associated with blunted cardiovascular, neuroendocrine and cognitive responses to acute mental stress. PloS One 7(6):e39143. doi:10.1371/journal.pone.0039143

Kaore SN, Amane HS, Kaore NM (2013) Citrulline: pharmacological perspectives and its role as an emerging biomarker in future. Fundam Clin Pharmacol 27(1):35-50. doi:10.1111/j.1472-8206. 2012.01059.x

Krug S, Kastenmüller G, Stückler F, Rist MJ, Skurk T, Sailer M, Raffler J, et al (2012) The dynamic range of the human metabolome revealed by challenges. FASEB J. doi:10.1096/fj. $11-198093$

Laus MF, Duarte Manhas Ferreira Vales L, Braga Costa TM, Sousa Almeida S (2011) Early postnatal protein-calorie malnutrition and cognition: a review of human and animal studies. Int $\mathbf{J}$ Environ Res Public Health 8(2):590-612. doi:10.3390/ ijerph8020590

Lawton KA, Berger A, Mitchell M, Milgram KE, Evans AM, Guo L, Hanson RW, Kalhan SC, Ryals JA, Milburn MV (2008) Analysis of the adult human plasma metabolome. Pharmacogenomics 9 (1744-8042 (Electronic) LA-eng PT-Comparative Study PTJournal Article SB-IM). Metabolon, Inc, 800 Capitola Dr. Suite 1, Durham, NC 27713, USA: 383-97. PM:18384253

Liang W, Lindeman JH, Menke AL, Koonen DP, Morrison M, Havekes LM, van den Hoek AM, Kleemann R (2014) Metabolically induced liver inflammation leads to NASH and differs 
from LPS- or IL-1 $\beta$-induced chronic inflammation. Lab Invest 94(5). Nature Publishing Group: 491-502. doi:10.1038/labin vest.2014.11

Lichtenstein L, Mattijssen F, de Wit NJ, Georgiadi A, Hooiveld GJ, van der Meer R, He Y et al (2010) Angptl4 protects against severe proinflammatory effects of saturated fat by inhibiting fatty acid uptake into mesenteric lymph node macrophages. Cell Metab 12(6):580-592. doi:10.1016/j.cmet.2010.11.002

Lusis AJ, Attie AD, Reue K (2008) Metabolic syndrome: from epidemiology to systems biology. Nat Rev Genet 9(11):819-830. doi:10.1038/nrg2468

Malaguarnera M, Gargante MP, Russo C, Antic T, Vacante M, Malaguarnera M, Avitabile T, Volti GL, Galvano F (2010) L-Carnitine supplementation to diet: a new tool in treatment of nonalcoholic steatohepatitis: a randomized and controlled clinical trial. Am J Gastroenterol 105(6):1338-1345. doi:10.1038/ ajg.2009.719

McCormack SE, McCarthy MA, Harrington SG, Farilla L, Hrovat MI, Systrom DM, Thomas BJ et al (2013) Effects of exercise and lifestyle modification on fitness, insulin resistance, skeletal muscle oxidative phosphorylation and intramyocellular lipid content in obese children and adolescents. Pediatr Obes. doi:10. 1111/j.2047-6310.2013.00180.x

Medina-Gomez G, Gray SL, Yetukuri L, Shimomura K, Virtue S, Campbell M, Curtis RK, et al (2007) PPAR gamma 2 prevents lipotoxicity by controlling adipose tissue expandability and peripheral lipid metabolism. PLoS.Genet 3(1553-7404 (Electronic) LA-eng PT-Comparative Study PT-Journal Article PTResearch Support, Non-U.S. Gov't RN-0 (Lipids) RN-0 (PPAR gamma) RN-11061-68-0 (Insulin) SB-IM). Department of Clinical Biochemistry, Histopathology, University of Cambridge/Addenbrooke's Hospital, Cambridge, United Kingdom: e64. PM:17465682

Menni C, Fauman E, Erte I, Perry JRB, Kastenmüller G, Shin S-Y, Petersen A-K et al (2013) Biomarkers for type 2 diabetes and impaired fasting glucose using a nontargeted metabolomics approach. Diabetes 62(12):4270-4276. doi:10.2337/db13-0570

Mihas C, Kolovou GD, Mikhailidis DP, Kovar J, Lairon D, Nordestgaard BG, Chye Ooi T et al (2011) Diagnostic value of postprandial triglyceride testing in healthy subjects: a metaanalysis. Curr Vasc Pharmacol 9(3):271-280

Musso G, Gambino R, Pacini G, De Michieli F, Cassader M (2009) Prolonged saturated fat-induced, glucose-dependent insulinotropic polypeptide elevation is associated with adipokine imbalance and liver injury in nonalcoholic steatohepatitis: dysregulated enteroadipocyte axis as a novel feature of fatty liver. Am J Clin Nutr 89(2):558-567. doi:10.3945/ajen.2008. 26720

Newgard CB, An J, Bain JR, Muehlbauer MJ, Stevens RD, Lien LF, Haqq AM, et al (2009) A branched-chain amino acid-related metabolic signature that differentiates obese and lean humans and contributes to insulin resistance. Cell Metab 9(4). Elsevier Ltd: 311-326. doi:10.1016/j.cmet.2009.02.002

Noland RC, Koves TR, Seiler SE, Lum H, Lust RM, Ilkayeva O, Stevens RD, Hegardt FG, Muoio DM (2009) Carnitine insufficiency caused by aging and overnutrition compromises mitochondrial performance and metabolic control. J Biol Chem 284(34):22840-22852. doi:10.1074/jbc.M109.032888

Osborn O, Olefsky JM (2012) The cellular and signaling networks linking the immune system and metabolism in disease. Nat Med 18(3). Nature Publishing Group: 363-374. doi:10.1038/nm.2627

Pellis L, Erk MJ, Ommen B, Bakker GCM, Hendriks HFJ, Cnubben NHP, Kleemann R et al (2011) Plasma metabolomics and proteomics profiling after a postprandial challenge reveal subtle diet effects on human metabolic status. Metabolomics. doi:10. 1007/s11306-011-0320-5
Phillips CM, Goumidi L, Bertrais S, Ferguson JF, Field MR, Kelly ED, Mehegan J et al (2010) Additive effect of polymorphisms in the IL-6, LTA, and TNF-alpha genes and plasma fatty acid level modulate risk for the metabolic syndrome and its components. J Clin Endocrinol Metab 95(3):1386-1394

Rutlegde AC, Adeli K (2007) Fructose and the metabolic syndrome: pathophysiology and molecular mechanisms. Nutr Rev 65(6):13-23. doi:10.1301/nr.2007.jun.S13

Shaham O, Wei R, Wang TJ, Ricciardi C, Lewis GD, Vasan RS, Carr SA, Thadhani R, Gerszten RE, Mootha VK (2008) Metabolic profiling of the human response to a glucose challenge reveals distinct axes of insulin sensitivity. Mol Syst Biol 4(214). EMBO and Nature Publishing Group: 214. doi:10.1038/msb.2008.50

Spégel P, Danielsson APH, Bacos K, Nagorny CLF, Moritz T, Mulder H, Filipsson K (2009) Metabolomic analysis of a human oral glucose tolerance test reveals fatty acids as reliable indicators of regulated metabolism. Metabolomics 6(1):56-66. doi:10.1007/ s11306-009-0177-z

Steptoe A, Dockray S, Wardle J (2009) Positive affect and psychobiological processes relevant to health. J Pers 77(6):1747-1776. doi:10.1111/j.1467-6494.2009.00599.x

Van Dijk SJ, Mensink M, Esser D, Feskens EJM, Müller M, Afman LA (2012) Responses to high-fat challenges varying in fat type in subjects with different metabolic risk phenotypes: a randomized trial. PloS One 7(7):e41388. doi:10.1371/journal.pone. 0041388

Van Ommen B, Keijer J, Kleemann R, Elliott R, Drevon CA, McArdle H, Gibney M, Müller M (2008) The challenges for molecular nutrition research 2: quantification of the nutritional phenotype. Genes Nutr 3(2):51-59. doi:10.1007/s12263-0080084-3

Van Ommen B, Keijer J, Heil SG, Kaput J (2009) Challenging homeostasis to define biomarkers for nutrition related health. Mol Nutr Food Res 53(July). RIKILT-Institute of Food Safety, Wageningen University and Research Centre, Wageningen, The Netherlands. ben.vanommen@tno.nl: 795-804. PM:19517455

Vance DE (2013) Phospholipid methylation in mammals: from biochemistry to physiological function. Biochim Biophys Acta. doi:10.1016/j.bbamem.2013.10.018

Virtue S, Vidal-Puig A (2010) Adipose tissue expandability, lipotoxicity and the metabolic syndrome-an allostatic perspective. Biochim Biophys Acta 1801(3). Elsevier B.V. 338-49. doi:10. 1016/j.bbalip.2009.12.006

Vis DJ, Westerhuis JA, Jacobs DM, van Duynhoven JPM, Wopereis S, van Ommen B, Hendriks MMWB, Smilde AK (2014) Analyzing metabolomics-based challenge tests. Metabolomics. doi:10.1007/s11306-014-0673-7

Wopereis S, Rubingh CM, van Erk MJ, Verheij ER, van Vliet T, Cnubben NHP, Smilde AK, van der Greef J, van Ommen B, Hendriks HFJ (2009) Metabolic profiling of the response to an oral glucose tolerance test detects subtle metabolic changes. PLoS ONE 4(2). Public Library of Science: e4525. doi:10.1371/ journal.pone. 0004525

Wopereis S, Wolvers D, van Erk M, Gribnau M, Kremer B, van Dorsten FA, Boelsma E et al (2013) Assessment of inflammatory resilience in healthy subjects using dietary lipid and glucose challenges. BMC Med Genom 6(1):44. doi:10.1186/1755-87946-44

Zhao X, Peter A, Fritsche J, Elcnerova M, Fritsche A, Häring H-U, Schleicher ED, Xu G, Lehmann R (2009) Changes of the plasma metabolome during an oral glucose tolerance test: is there more than glucose to look at? Am J Physiol Endocrinol Metab 296(2):E384-E393. doi:10.1152/ajpendo.90748.2008 